DOE/ER/40762-163

U. of MD PP\#99-038

\title{
Discretization Errors and Rotational Symmetry: The Laplacian Operator on Non-Hypercubical Lattices
}

\author{
Chi-Keung Chow \\ Department of Physics, University of Maryland, College Park, 20742-4111.
}

(November 17, 2017)

\begin{abstract}
Discretizations of the Laplacian operator on non-hypercubical lattices are discussed in a systematic approach. It is shown that order $a^{2}$ errors always exist for discretizations involving only nearest neighbors. Among all lattices with the same density of lattice sites, the hypercubical lattices always have errors smaller than other lattices with the same number of spacetime dimensions. On the other hand, the four dimensional checkerboard lattice (also known as the Celmaster lattice) is the only lattice which is isotropic at order $a^{2}$. Explicit forms of the discretized Laplacian operators on root lattices are presented, and different ways of eliminating order $a^{2}$ errors are discussed.
\end{abstract}

Typeset using REVTEX 


\section{INTRODUCTION}

Lattice simulation is an invaluable tool in our studies of nonperturbative aspects of quantum field theories. By the discretization of the spacetime continuum, the original system with infinitely many degrees of freedom is approximated by one with only a large but finite number of degrees of freedom, which can be studied numerically. In practice, the lattice has almost always been chosen as hypercubical since the original paper by Wilson [1], although some "uncanonical" choices has also been discussed. Four-dimensional random lattices were discussed by Christ et. al. in Ref. [2 - The body-centered hypercubical lattices in four dimensions had been first studied by Celmaster [5 [7 and subsequently in [8 11]. Almost at the same time four dimensional simplicial lattices were studied in [12 16]. (An interesting comparison between the last two uncanonical lattices can be found in Ref. [17.)

Let's recall the definition of a lattice. A set of vectors $\left\{B_{i}\right\}(1 \leq i \leq n)$ in $m$-dimensional space $\mathbf{R}^{m}$ is linearly independent if

$$
\sum_{i=1}^{n} x_{i} B_{i}=0 \quad \text { iff } \quad x_{i}=0 \quad \forall i .
$$

With such a set of linearly independent basis vectors $\left\{B_{i}\right\}$ one can define a lattice as below.

An $n$-dimensional lattice $\Lambda_{n}$ is a subset of $\mathbf{R}^{m}(n \leq m)$ such that

$$
\Lambda_{n}=\left\{X=\sum_{i=1}^{n} z_{i} B_{i}: z_{i} \in \mathbf{Z}\right\}
$$

The lattice always contains the origin and is symmetric under reflection $X \rightarrow-X$ in $\mathbf{R}^{m}$. Notice that we have not required that $n=m$ as some lattices (like the triangular lattice in two dimensions) can be more conveniently represented when embedded in some higher dimensional spaces. Also note that, under this definition, random lattices are not lattices. Still, such a definition encompasses a huge set of lattices, including extremely skewed ones whose unitcells are long narrow parallelograms or parallelohedra. However, such highly asymmetric lattices are rarely used for simulations. Instead, physicists always use lattices 
which are "highly symmetric" (we will give a mathematically precise meaning to this phrase later). In particular, there is a class of lattices called the "root lattices", which contains most of the "highly symmetric" lattices, at least in lower dimensions $n \leq 4$. While there is no real justification in neglecting the lattices which do not belong to this class, root lattices are so well studied that they provide a natural starting point for our investigation. As we will see, there are exactly three root lattices in four dimensions, namely the hypercubical, the body-centered hypercubical and the simplicial lattices円, i.e., exactly the cases which have been studied.

It is important to compare the relative merits of these different lattices in simulations. Several papers mentioned above attempted to answer this question by simulating the same physical system on different lattices and comparing the results. These comparisons are very directly relevant to the practical applications of lattice simulations, but theoretically they are "unclean" in the sense that they are a priori sensitive to the physical problems in question, the actual algorithms used, and other non-geometrical factors. This paper attempts to study the same problem in a complementary way. We will obtain a general prescription on how to construct the Laplacian operator, the simplest possible differential operator, on any lattice. While the Laplacian operator is not directly relevant for lattice QCD, it is used in lattice simulations of strongly coupled scalar $\phi^{4}$ theories, and it is logical to study the Laplacian case thoroughly before tackling the more complicated cases of the Dirac and the plaquette operators. By studying the discretization errors of the Laplacian operators mathematically, one can parameterize these errors in ways which depend only on the geometrical properties of the lattices. The questions we will be addressing include:

- Are there any lattices on which the discretizations of the Laplacian operator on the nearest neighbors are free of $\mathcal{O}\left(a^{2}\right)$ errors?

- What are the merits and demerits of our canonical choice - the simple hypercubical

\footnotetext{
${ }^{1}$ This fact has been observed in Ref. [12].
} 
lattice?

- What is the role of symmetry?

- In general, how do we eliminate the $\mathcal{O}\left(a^{2}\right)$ errors? (Answer: by involving sites which are not nearest neighbors.) Is it always possible?

- In a given dimension, does the lattice which has the smallest $\mathcal{O}\left(a^{2}\right)$ errors still work best after the $\mathcal{O}\left(a^{2}\right)$ errors are cancelled?

In this paper, we will try to provide answers to all these questions. The approach is "clean" in the sense that all the effects of algorithms or choices of physics problems are disentangled. On the other hand, it must be admitted that its relevance to practical lattice simulations is not immediate. Roughly speaking, this paper is more about lattice field theory than about lattice simulations, and the theorems obtained should be regarded as general "rules of thumb" instead of infallible laws in practice.

This paper is organized as follows: In Section II we present a prescription (one shell discretization) for approximating the Laplacian on a lattice. Root lattices are defined in Section III, and the explicit forms of the discretized Laplacian operators on root lattices are presented in Section IV. Order $a^{2}$ errors are studied in Sections V and VI, while the issue of anisotropy is discussed in Section VII. In Section VIII a general prescription (two shell discretization) for eliminating the order $a^{2}$ errors is presented, and the order $a^{4}$ errors in this scheme are estimated in Section IX, followed by the conclusion in Section X.

\section{LAPLACIAN ON A LATTICE}

On hypercubical lattices, the discretization of the Laplacian operator is usually constructed through the trapezoidal rule applied on each lattice direction. For example, the second derivative in the $x$ direction of an arbitrary function $\phi$ at the origin is

$$
a^{2} \partial_{x}^{2} \phi(0)=\phi(a \hat{x})-2 \phi(0)+\phi(-a \hat{x})+\ldots,
$$

and by summing up over all lattice directions, 


$$
\begin{aligned}
a^{2} \partial^{2} \phi(0) & =\sum_{\hat{\mu}}(\phi(a \hat{\mu})-2 \phi(0)+\phi(-a \hat{\mu}))+\ldots \\
& =\left\{\sum_{\hat{\mu}}\left(\exp \left(a \partial_{\mu}\right)-2+\exp \left(-a \partial_{\mu}\right)\right)\right\} \phi(0)+\ldots,
\end{aligned}
$$

where the $\hat{\mu}$ 's are the unit vectors along the lattice directions.

The construction above depends so heavily on the existence of a set of mutually orthogonal lattice vectors $\{\hat{\mu}\}$ that it cannot be naturally generalized to non-hypercubical lattices. (Try the two dimensional triangular lattice, for instance.) To obtain a construction which is equally applicable to hypercubical and non-hypercubical lattices, we will make use of the coordinate-independent definition of the Laplacian operator. Recall that when $\phi$ satisfies the $n$-dimensional Laplace equation $\partial^{2} \phi=0$, the value of $\phi$ at any point is the average of its value on an $(n-1)$-dimensional hypersphere of arbitrary radius centered at the point in question.

$$
\phi(0)=\int_{S^{n-1}} d \mu_{n-1} \phi(X),
$$

where $d \mu_{n-1}$ is the unit measure on $S^{n-1}$.

$$
\int_{S^{n-1}} d \mu_{n-1} \equiv \int_{S^{n-1}} d \Omega_{n-1} \frac{\Gamma(n / 2)}{2 \pi^{n / 2}}=1
$$

So one can take the Laplacian $\partial^{2} \phi$ as an indicator of how much the equality Eq. (2.3) is violated. Just from dimensional analysis one knows that

$$
\partial^{2} \phi(0)=N \lim _{r \rightarrow 0}\left(\int_{S^{n-1}} d \mu_{n-1} \phi(X)-\phi(0)\right) / r^{2}
$$

where $r$ is the radius of the hypersphere $S^{n-1}$, and $N$ is a proportionality constant one can easily determine to be $2 n$.

\footnotetext{
${ }^{2}$ The value of $N$ can be determined by considering the following electrostatic system satisfying the Poisson equation $\partial^{2} \phi=\rho$ in $n$ dimensions. Consider a ball of constant charge density $\rho$ and radius $r$. One can easily see that the potential difference between the surface and the center of the ball is $\rho r^{2} / 2 n$. Substitute this back into Eq. (2.5) and $N$ is found to be $2 n$.
} 
On a lattice, however, $\phi$ is only defined on discrete lattice sites, and one can at best approximate the integral with the sum of $\phi$ over discrete points. It is useful to introduce some formalism here and define a shell $\mathcal{S}_{r}$ of lattice sites with radius $r>0$ as the set of all sites with $\|X\|=r$. Obviously these $\mathcal{S}_{r}$ 's are non-empty only for discrete values of the $r$ 's, and the smallest of these values, $\rho$, is the separation between each lattice site and its nearest neighbors and is usually called the packing diameter of the lattice. We will call $\mathcal{S}_{\rho}$ the first shell, and its cardinality, i.e., the number of nearest neighbors, denoted by $\tau$, is called the kissing number ${ }^{3}$. Now we approximate the integral of $\phi$ over $S^{n-1}$ by its average $\Sigma$ over the first shell

$$
\Sigma \phi(0)=\frac{1}{\tau} \sum_{X_{k} \in \mathcal{S}_{\rho}} \phi\left(X_{k}\right)
$$

where $\Sigma$ is defined as an operator over $\phi$, which can also be expressed in terms of the partial derivatives.

$$
\Sigma \phi(0)=\left\{\frac{1}{\tau} \sum_{X_{k} \in \mathcal{S}_{\rho}} \exp \left(X_{k} \cdot \partial\right)\right\} \phi(0)
$$

The corresponding approximation of the Laplacian operator is

$$
D^{2} \phi(0)=2 n\left(\frac{1}{\tau} \sum_{X_{k} \in \mathcal{S}_{\rho}} \phi\left(X_{k}\right)-\phi(0)\right) / \rho^{2}=2 n(\Sigma-1) \phi(0) / \rho^{2}
$$

The nonlocal operator $D^{2}$ defined above, called the one shell discretization of the Laplacian operator, is the main object of this study. How good is $D^{2}$ as an approximation of $\partial^{2}$ ? Note that

$$
\begin{aligned}
\rho^{2} D^{2} \phi(0) / 2 n & =\frac{1}{\tau} \sum_{X_{k} \in \mathcal{S}_{\rho}} \phi\left(X_{k}\right)-\phi(0)=\frac{1}{\tau} \sum_{X_{k} \in \mathcal{S}_{\rho}}\left(\phi\left(X_{k}\right)-\phi(0)\right) \\
& =\left\{\frac{1}{\tau} \sum_{X_{k} \in \mathcal{S}_{\rho}}\left(\exp \left(X_{k} \cdot \partial\right)-1\right)\right\} \phi(0) .
\end{aligned}
$$

\footnotetext{
${ }^{3}$ Also known to chemists as the contact number or the coordination number, and to historians as the Newton number, after Isaac Newton who studied the problem in three dimensions.
} 
So the expression inside the braces is a derivative representation of the nonlocal operator $\rho^{2} D^{2} / 2 n$. By expanding the exponential, which is equivalent to a Taylor expansion in $\rho$, one can express $\rho^{2} D^{2} / 2 n$ in terms of powers of the derivative operator. Now

$$
\exp \left(X_{k} \cdot \partial\right)=1+X_{k} \cdot \partial+\frac{1}{2}\left(X_{k} \cdot \partial\right)^{2}+\frac{1}{6}\left(X_{k} \cdot \partial\right)^{3}+\mathcal{O}\left(\rho^{4}\right)
$$

The order $\rho^{0}$ term is just unity, which is cancelled by the " -1 " coming from the $\phi(0)$ term in Eq. (2.8). The order $\rho^{1}$ term is proportional to

$$
\left(\sum_{X_{k} \in \mathcal{S}_{\rho}} X_{k}\right) \cdot \partial=0
$$

due to the reflection symmetry of the lattice. Similarly, all odd powers of $\rho$ vanish due to

reflection symmetry. On the other hand, the $\rho^{2}$ term is $\frac{1}{2} \sum_{k}\left(X_{k} \cdot \partial\right)^{2}$ which is in general not a Laplacian unless the set of nearest neighbors $\mathcal{S}_{\rho}$ has vanishing quadrapole moment. So we have the theorem:

The one shell discretization $D^{2}$ of the Laplacian operator over the first shell $\mathcal{S}_{\rho}$ is equal to the Laplacian operator $\partial^{2}$ itself in leading order if and only if the quadrapole moment of $\mathcal{S}_{\rho}$ vanishes.

It must be emphasized that the theorem above applies only for one shell discretizations as defined in Eq. (2.8). In general one may give different weights to different lattice sites in $\mathcal{S}_{\rho}$ in the sum in Eq. (2.8) or even extend the sum to sites which are not nearest neighbors, and the theorem does not apply to such constructions. However, such alternative discretization schemes contain many free arbitrary parameters (as in the weights) and they are clearly beyond the scope of this paper.

\section{ROOT LATTICES}

We have seen that the one shell discretization method provides a good approximation of the Laplacian operator as long as the nearest shell of neighbors has vanishing quadrapole 
moment. Unfortunately, the author is not aware of any classification of the lattices satisfying this condition. However, it is well known that root lattices have been completely classified, and all of them have vanishing quadrapole moment. Moreover, both the body-centered hypercubical and the simplicial lattices fall into this class, and so does the usual hypercubical lattice. So it is useful to study these root lattices closely.

A root lattice is one whose basis vectors can all be chosen to be nearest neighbors and the angles between any two of them are either $\pi / 2$ or $2 \pi / 3$. In other words, when the basis vectors are scaled to have unit length, the inner products of any two of them are either 0 or $-\frac{1}{2}$.

This definition depends solely on the relative directions of the nearest neighbors (as opposed to definitions in textbooks on Lie groups, which may involve next-to-nearest neighbors) is more useful for the study of one shell discretizations, which also just depend on the first shell.

The classification theorem for root lattices is as follows:

An n-dimensional root lattice is either isometric to one of the Cartan-Dynkin root lattices $Z_{n}, A_{n}, D_{n}$ and $E_{n}$ for some value of $a$, or is an outer product of several of them with the same $a$.

The Cartan-Dynkin lattices $Z_{n}, A_{n}$ and $D_{n}$ are defined as below for any $n \in \mathbf{Z}$ :

$$
\begin{aligned}
& Z_{n} \equiv\left\{X=\left(x_{1}, \ldots, x_{n}\right) \quad \in a \mathbf{Z}^{n}\right\}, \\
& A_{n} \equiv\left\{X=\left(x_{1}, \ldots, x_{n}, x_{n+1}\right) \in a \mathbf{Z}^{n+1} ; \sum_{k} x_{k}=0\right\}, \\
& D_{n} \equiv\left\{X=\left(x_{1}, \ldots, x_{n}\right) \quad \in a \mathbf{Z}^{n} ; \sum_{k} x_{k} \in 2 a \mathbf{Z}\right\},
\end{aligned}
$$

These representations have the nice features that the coordinates of the lattice sites are always integer multiples of the lattice spacing $a$, and each lattice is symmetric under permutations of the coordinates. It is obvious that $Z_{n}$ are the square, cubic and hypercubical 
lattices for $n=2,3$ and 4 respectively. The simplicial lattices $A_{n}$ are for $n=2$ the hexagonal lattices, for $n=3$ the face-centered cubic lattices, and for $n=4$ the four dimensional simplicial lattices studied in Ref. [12 [16]. The checkerboard lattices $D_{n}$ are also square lattices for $n=2\left(D_{2} \simeq Z_{2}\right)$, face-centered cubic lattices for $n=3\left(D_{3} \simeq A_{3}\right)$ and Celmaster lattices [5 7] for $n=4$. Lastly, the $E_{n}$ lattices are defined only for $n=6,7$ or 8 . Since we are mainly interested in lattices in low dimensions, we will not discuss $E_{n}$ and refer the interested reader to Ref. [18] instead.

Several questions immediately come to mind. Firstly, are the $A_{n}$ and $D_{n}$ really the same Cartan-Dynkin root lattices one uses for the representations of Lie groups? What is $Z_{n}$ in that language, and where are the $B_{n}, C_{n}$, and the exceptional groups? The first question can be easily answered by evaluating the angles between the basis vectors of $A_{n-1}$ :

$$
\left(x_{1}, \ldots, x_{n}\right)=(1,-1,0, \ldots, 0),(0,1,-1,0, \ldots, 0), \ldots,(0, \ldots, 0,1,-1)
$$

where each vector is rotated by an angle $2 \pi / 3$ from its predecessor as described by the $A_{n}$ Dynkin diagrams. The $D_{n}$ lattice has one more basis vector than $A_{n-1}$, namely $(-1,-1,0, \ldots, 0)$, which is orthogonal to the first basis vector of $A_{n-1}$ but makes an angle of $2 \pi / 3$ with the second, exactly as decreed by the $D_{n}$ Dynkin diagrams. Lastly, the $Z_{n}$ lattice also has one more basis vector than $A_{n-1}$, namely $(-1,0, \ldots, 0)$, which makes an angle $3 \pi / 4$ with the first basis vector of $A_{n-1}$, is orthogonal to the rest, and is shorter than the rest of the basis vectors by a factor of $1 / \sqrt{2}$, i.e., it has exactly the properties of the "double bond" of the Dynkin diagram of $B_{n}$. In other words, $Z_{n}$ is the root lattice of the Lie group $B_{n}$.

The last exercise illustrates the fact that a given root lattice can be spanned by different sets of basis vectors (and hence different Lie groups), and the choice most convenient for the study of Lie groups is not necessarily the most convenient for our purposes. As we have seen, the set of nearest neighbors $\mathcal{S}_{\rho}$ plays a special role in our discussion. As a result, we have

\footnotetext{
${ }^{4}$ Readers who are not familiar with the Cartan-Dynkin classification scheme for Lie groups can consult Slansky [19] and Georgi [20].
} 
chosen to define root lattices in a way which depends only on the relative directions of the nearest neighbors. The unique Lie group defined by the nearest neighbors on a given root lattice is always simply laced (no multiple bonds, all basis vectors have the same length). For $A_{n}$ it is $\mathrm{SU}(n+1)$, for $D_{n}$ it is $\mathrm{SO}(2 n)$ and for $Z_{n}$ it is $\mathrm{SU}(2)^{n}$ (not $\mathrm{SO}(2 n-1)$ ).

The remaining Dynkin diagrams $\left(C_{n}, F_{4}\right.$ and $\left.G_{2}\right)$ are all multiply laced (with multiple bonds, and basis vectors of different lengths). One can easily check that the $C_{n}$ and $D_{n}$ groups have the same root lattices, but the former is doubly laced while the latter is simply laced. Similarly, the simply laced counterparts of $G_{2}$ and $F_{4}$ are $A_{2}$ and $D_{4}$ respectively?.

So we have clarified the relationship between the Lie groups (which have little to do with this paper) and the root lattices (our real concern). The reader should be warned that, for the purposes of this paper, it is advisable to temporarily forget about the Lie group structures of the lattices and regard the root lattices as geometrical constructions in their own right.

\section{DISCRETIZED LAPLACIAN OPERATORS ON ROOT LATTICES}

In this section, we will give explicit formulae for $\Sigma$ and $D^{2}$ on root lattices $Z_{n}, A_{n}$ and $D_{n}$. Recall that, since all lattices are symmetrical under reflection with respect to the origin $\left(X_{m} \rightarrow-X_{m}\right.$ for all $m, m$ being the dimensionality of the coordinate space, i.e., $n$ for $Z_{n}$ and $D_{N}$ and $n+1$ for $A_{n}$ ), in the Taylor expansion of $\Sigma$, only terms with even powers of $\rho$ survive. Hence

$$
\Sigma=\frac{1}{\tau} \sum_{X_{k} \in \mathcal{S}_{\rho}} \exp \left(X_{k} \cdot \partial\right) \equiv \mathcal{O}_{0}+a^{2} \mathcal{O}_{2}+a^{4} \mathcal{O}_{4}+a^{6} \mathcal{O}_{6}+\ldots
$$

\footnotetext{
${ }^{5}$ This is why we avoid calling the hypercubical lattice $B_{n}$ — to emphasize that we are studying the root lattice, not the Lie group.

${ }^{6}$ The authors of Ref. [9 11] actually referred to the Celmaster lattice $D_{4}$ as the $F_{4}$ lattice.
} 
The operators $\mathcal{O}_{l}$ are $l$-th order derivatives, their forms constrained by the symmetries of the lattices. As mentioned before, all three root lattices defined in Eq. (3.1) are invariant under permutations of the coordinates. Moreover, $Z_{n}$ and $D_{n}$ are also symmetric under reflection of individual coordinates $\left(X_{m} \rightarrow-X_{m}\right.$ for a given $\left.m\right)$. These symmetry constraints limit the number of possible operators appearing at each order.

To enumerate the number of possible operators appearing at each order, the following definitions are useful.

$$
\begin{aligned}
\mathcal{B}_{j_{1} j_{2} \ldots j_{p}}^{(m)} & =\sum_{1 \leq i_{1} \neq i_{2} \neq \ldots \neq i_{p} \leq m} \partial_{i_{1}}^{j_{1}} \partial_{i_{2}}^{j_{2}} \ldots \partial_{i_{p}}^{j_{p}}, \quad \text { all } j \text { 's even, } \\
\mathcal{A}_{j_{1} j_{2} \ldots j_{p}}^{(m)} & =\sum_{1 \leq i_{1} \neq i_{2} \neq \ldots \neq i_{p} \leq m} \partial_{i_{1}}^{j_{1}} \partial_{i_{2}}^{j_{2}} \ldots \partial_{i_{p}}^{j_{p}}, \quad \text { some } j \text { 's odd. }
\end{aligned}
$$

The definitions of the even operators $\mathcal{B}$ and the odd operators $\mathcal{A}$ are actually identical except that $\mathcal{A}$ may contain odd powers of $\partial_{i}$, which is not invariant under reflection of coordinate $x_{i}$. As a result, $\mathcal{O}_{l}$ for $Z_{n}$ and $D_{n}$ contains only terms like $\mathcal{B}_{j_{1} j_{2} \ldots j_{p}}^{(n)}$ with $\sum_{p} j_{p}=l$ but no $\mathcal{A}$ terms. On the other hand, for $A_{n}$, both $\mathcal{B}_{j_{1} j_{2} \ldots j_{p}}^{(n+1)}$ and $\mathcal{A}_{j_{1} j_{2} \ldots j_{p}}^{(n+1)}$ contribute.

It is easy to see that $\mathcal{O}_{0}=1$ for any lattice. There are two operators which appear at order $a^{2}$, namely

$$
\begin{aligned}
& \mathcal{B}_{2}^{(m)}=\sum_{1 \leq i \leq m} \partial_{i}^{2}, \\
& \mathcal{A}_{11}^{(m)}=\sum_{1 \leq i \neq j \leq m} \partial_{i} \partial_{j} .
\end{aligned}
$$

As mentioned before, for $Z_{n}$ and $D_{n}, \mathcal{A}$ does not contribute and $\mathcal{O}_{2}$ must be proportional to the even operator $\mathcal{B}_{2}^{(m)}$, which is exactly the Laplacian operator in $m$ dimensions, and $m=n$ for $Z_{n}$ and $D_{n}$. On the other hand, for $A_{n}, \mathcal{O}_{2}$ cannot be simply proportional to $\mathcal{B}_{2}^{(m)}$, as we want something proportional to the Laplacian operator in $n$, not $m=n+1$, dimensions. However, for $A_{n}$ the odd operator $\mathcal{A}_{11}^{(m)}$ does contribute, and as we will see below, exactly in the right amount to give the Laplacian operator in an $n$ dimensional subspace.

When one goes to order $a^{4}$, more operators appear.

$$
\mathcal{B}_{4}^{(m)}=\sum_{1 \leq i \leq m} \quad \partial_{i}^{4}
$$




$$
\begin{array}{cl}
\mathcal{B}_{22}^{(m)}=\sum_{1 \leq i \neq j \leq m} & \partial_{i}^{2} \partial_{j}^{2}, \\
\mathcal{A}_{31}^{(m)}=\sum_{1 \leq i \neq j \leq m} \quad \partial_{i}^{3} \partial_{j}, \\
\mathcal{A}_{211}^{(m)}=\sum_{1 \leq i \neq j \neq k \leq m} \partial_{i}^{2} \partial_{j} \partial_{k}, \\
\mathcal{A}_{1111}^{(m)}=\sum_{1 \leq i \neq j \neq k \neq l \leq m} \partial_{i} \partial_{j} \partial_{k} \partial_{l} .
\end{array}
$$

As before, the odd operators only contribute in the $A_{n}$ case while the even ones in general may contribute for all three classes of root lattices.

After discussing the generalities, we will move on to specific cases and calculate $\Sigma$, and hence $D^{2}=2 n(\Sigma-1) / \rho^{2}$, for all three classes of root lattices. We will also list the kissing numbers $\tau$, packing diameters $\rho$ and densities of lattice sites $\delta$ of the lattices. Many of the properties listed below are extracted from the excellent book by Conway and Sloane [18], although the definitions of $\rho$ and $\delta$ here are different from those in the book.

\section{A. The Hypercubical Lattice $Z_{n}$}

The hypercubical lattice $Z_{n}$, defined as

$$
Z_{n} \equiv\left\{X=\left(x_{1}, \ldots, x_{n}\right) \in a \mathbf{Z}^{n}\right\}
$$

has kissing number $\tau=2 n$, packing diameter $\rho=a$ and density $\delta=1 / a^{n}$. One can easily evaluate $\Sigma$, which takes the form

$$
\Sigma=1+\frac{1}{2 ! n} a^{2} \mathcal{B}_{2}^{(n)}+\frac{1}{4 ! n} a^{4} \mathcal{B}_{4}^{(n)}+\mathcal{O}\left(a^{6}\right) .
$$

One can easy show that, due to the orthogonal nature of $Z_{n}$,

$$
\mathcal{O}_{l}=\frac{1}{l ! n} \sum_{1 \leq i \leq n} \partial_{i}^{l}
$$

Lastly, the one shell discretization $D^{2}$ is

$$
D^{2}=\mathcal{B}_{2}^{(n)}+\frac{2}{4 !} a^{2} \mathcal{B}_{4}^{(n)}+\ldots=\partial^{2}+\frac{1}{12} a^{2} \sum_{1 \leq i \leq n} \partial_{i}^{4}+\mathcal{O}\left(a^{4}\right),
$$

and as predicted, $D^{2}=\partial^{2}$ in the leading order. 


\section{B. The Checkerboard Lattice $D_{n}$}

The checkerboard lattice $D_{n}$, defined as

$$
D_{n} \equiv\left\{X=\left(x_{1}, \ldots, x_{n}\right) \in a \mathbf{Z}^{n} ; \sum_{k} x_{k} \in 2 a \mathbf{Z}\right\},
$$

has kissing number $\tau=2 n(n-1)$ and packing diameter $\rho=\sqrt{2} a$. Only half of the lattice sites of $Z_{n}$ is occupied in $D_{n}$, so its density $\delta=1 / 2 a^{n}$ is half of that of $Z_{n}$. It is straightforward to calculate $\Sigma$,

$$
\Sigma=1+\frac{2}{2 ! n} a^{2} \mathcal{B}_{2}^{(n)}+\frac{2}{4 ! n} a^{4} \mathcal{B}_{4}^{(n)}+\frac{6}{4 ! n(n-1)} a^{4} \mathcal{B}_{22}^{(n)}+\mathcal{O}\left(a^{6}\right),
$$

and bearing in mind that now $\rho^{2}=2 a^{2}$,

$$
\begin{aligned}
D^{2} & =\mathcal{B}_{2}^{(n)}+\frac{2}{4 !} a^{2} \mathcal{B}_{4}^{(n)}+\frac{6}{4 !(n-1)} a^{2} \mathcal{B}_{22}^{(n)}+\mathcal{O}\left(a^{4}\right) \\
& =\partial^{2}+\frac{1}{12} a^{2} \sum_{1 \leq i \leq n} \partial_{i}^{4}+\frac{1}{4(n-1)} a^{2} \sum_{1 \leq i \neq j \leq n} \partial_{i}^{2} \partial_{j}^{2}+\mathcal{O}\left(a^{4}\right) .
\end{aligned}
$$

Again, we verify that $D^{2}=\partial^{2}$ in the leading order 7 .

The four dimensional checkerboard lattice $D_{4}$ is of special importance as it has been extensively studied by Celmaster [5 [0] and is usually known as the Celmaster, or bodycentered hypercubical lattice.

$$
D_{4}^{*} \equiv\left\{X=\left(x_{1}, x_{2}, x_{3}, x_{4}\right) \in a \mathbf{Z}^{4} \cup a\left(\mathbf{Z}^{4}+\left(\frac{1}{2}, \frac{1}{2}, \frac{1}{2}, \frac{1}{2}\right)\right)\right\},
$$

i.e., the coordinates are either all integers or all half integers. The Celmaster lattice $D_{4}^{*}$ is actually the dual, or reciprocal lattice, of $D_{4}$. Reciprocal lattices are in general quite different: in three dimensions, the face-centered cubic and body-centered cubic lattices are reciprocal, and are not similar at all. In this special case, however, $D_{4}$ and $D_{4}^{*}$ are actually similar to each other. First note that both have 24 sites in the first shell:

$$
\begin{array}{ll}
D_{4}: & ( \pm 1, \pm 1,0,0)+\text { perm.; } \\
D_{4}^{*}: & ( \pm 1,0,0,0)+\text { perm. }, \quad\left( \pm \frac{1}{2}, \pm \frac{1}{2}, \pm \frac{1}{2}, \pm \frac{1}{2}\right)+\text { perm. }
\end{array}
$$

\footnotetext{
7 This discretization is equivalent to that used in Ref. 9 11]
} 
and 24 sites on the second shell

$$
\begin{array}{ll}
D_{4}: & ( \pm 2,0,0,0)+\text { perm. }, \quad( \pm 1, \pm 1, \pm 1, \pm 1)+\text { perm. } \\
D_{4}^{*}: & ( \pm 1, \pm 1,0,0)+\text { perm. }
\end{array}
$$

It is clear that the first shell of $D_{4}$ is exactly the second shell of $D_{4}^{*}$ and the second shell of $D_{4}$ is the first shell of $D_{4}^{*}$ scaled up by a factor of 2 . One can actually show that one can start out with $D_{4}$, perform a global rotation and overall rescaling by a factor of $1 / \sqrt{2}$ and end up with $D_{4}^{*}$. So $D_{4}$ and $D_{4}^{*}$ are geometrically similar in the sense that similar triangles are similar — they can be mapped onto each other through global rotation and overall scaling. As we will see below, the first and second shell of $D_{4}$ being similar will be important when we try to cancel $\mathcal{O}\left(a^{2}\right)$ errors by going beyond one shell discretization.

\section{The Simplicial Lattice $A_{n}$}

Lastly, we will discuss the simplicial lattice $A_{n}$, which is the hardest to study as it is embedded in an $n+1$-dimensional coordinate space.

$$
A_{n} \equiv\left\{X=\left(x_{1}, \ldots, x_{n}, x_{n+1}\right) \in a \mathbf{Z}^{n+1} ; \sum_{k} x_{k}=0\right\}
$$

it has kissing number $\tau=n(n+1)$, packing diameter $\rho=\sqrt{2} a$, and density $\delta=1 / \sqrt{n+1} a^{n}$, and

$$
\begin{aligned}
\Sigma=1 & +\frac{2}{2 !(n+1)} a^{2} \mathcal{B}_{2}^{(n+1)}+\frac{-2}{2 ! n(n+1)} a^{2} \mathcal{A}_{11}^{(n+1)} \\
& +\frac{2}{4 !(n+1)} a^{4} \mathcal{B}_{4}^{(n+1)}+\frac{6}{4 ! n(n+1)} a^{4} \mathcal{B}_{22}^{(n+1)}+\frac{-6}{4 ! n(n+1)} a^{4} \mathcal{A}_{31}^{(n+1)}+\mathcal{O}\left(a^{6}\right),
\end{aligned}
$$

without any terms proportional to $\mathcal{A}_{211}$ or $\mathcal{A}_{1111}$. One can then read off $D^{2}$, which takes a rather complicated form.

\footnotetext{
8 The easiest way to show this is by observing that $Z_{n+1}$ has density $1 / a^{n+1}$, and the $A_{n}$-like sublattices $\left\{X \in a \mathbf{Z}^{n+1} ; \sum_{k} x_{k}=C\right\}$ where $C$ is a fixed integer, have density $\sqrt{n+1} / a$ in $Z_{n+1}$. The quotient of the two gives the density of $A_{n}$.
} 


$$
\begin{aligned}
D^{2}= & \frac{n}{(n+1)} \mathcal{B}_{2}^{(n+1)}-\frac{1}{(n+1)} \mathcal{A}_{11}^{(n+1)} \\
& \quad+\frac{2 n}{4 !(n+1)} a^{2} \mathcal{B}_{4}^{(n+1)}+\frac{6 n}{4 ! n(n+1)} a^{2} \mathcal{B}_{22}^{(n+1)}-\frac{6 n}{4 ! n(n+1)} a^{2} \mathcal{A}_{31}^{(n+1)}+\mathcal{O}\left(a^{4}\right) \\
= & \frac{n}{(n+1)} \sum_{1 \leq i \leq n+1} \partial_{i}^{2}-\frac{1}{(n+1)} \sum_{1 \leq i \neq j \leq n+1} \partial_{i} \partial_{j} \\
& \quad+\frac{n}{12(n+1)} a^{2} \sum_{1 \leq i \leq n+1} \partial_{i}^{4}+\frac{1}{4(n+1)} a^{2} \sum_{1 \leq i \neq j \leq n+1} \partial_{i}^{2} \partial_{j}^{2}-\frac{1}{4(n+1)} a^{2} \sum_{1 \leq i \neq j \leq n+1} \partial_{i}^{3} \partial_{j}^{1}+\mathcal{O}\left(a^{4}\right) .
\end{aligned}
$$

Does the sum of the first two terms give the Laplacian operator in the $n$-dimensional subspace described by $\sum x_{i}=0$ ? Note the following relation between the Laplacian operators in $n$ and $n+1$ dimensions.

$$
\left.\partial^{2}\right|_{\mathbf{R}^{n+1}}=\left.\partial^{2}\right|_{\mathbf{R}^{n}}+\partial_{\bar{x}}^{2}
$$

where $\bar{x}=(1,1, \ldots, 1) / \sqrt{n+1}$ is the unit normal vector of the subspace $\sum x_{i}=0$. One can easily check that

$$
\partial_{\bar{x}}^{2}=\left(\sum_{1 \leq i \leq n+1} \partial_{i}^{2}+\sum_{1 \leq i \neq j \leq n+1} \partial_{i} \partial_{j}\right) /(n+1)
$$

and hence

$$
\left.\partial^{2}\right|_{\mathbf{R}^{n}}=\left.\partial^{2}\right|_{\mathbf{R}^{n+1}}-\partial_{\bar{x}}^{2}=\frac{n}{(n+1)} \sum_{1 \leq i \leq n+1} \partial_{i}^{2}-\frac{1}{(n+1)} \sum_{1 \leq i \neq j \leq n+1} \partial_{i} \partial_{j}
$$

exactly as advertised.

\section{DISCRETIZATION ERRORS}

We have seen that $D^{2}$ does reproduce $\partial^{2}$ exactly in leading order for all three classes of root lattices we have studied. In this section, we will analyze the order $a^{2}$ errors of $D^{2}$, which we will denote by $E$ and which is related to $\mathcal{O}_{4}$ by

$$
E=2 n \mathcal{O}_{4} a^{4} / \rho^{2}
$$

Its forms for all three classes of root lattices are summarized below. 


$$
\begin{aligned}
& Z_{n}: E=\frac{1}{4 !} a^{2} 2 \mathcal{B}_{4}^{(n)} \\
& D_{n}: E=\frac{1}{4 !} a^{2}\left(2 \mathcal{B}_{4}^{(n)}+\frac{6}{n-1} \mathcal{B}_{22}^{(n)}\right) \\
& A_{n}: E=\frac{1}{4 !} a^{2}\left(\frac{2 n}{n+1} \mathcal{B}_{4}^{(n+1)}+\frac{6 n}{n(n+1)} \mathcal{B}_{22}^{(n+1)}-\frac{6 n}{n(n+1)} \mathcal{A}_{31}^{(n+1)}\right) .
\end{aligned}
$$

At first sight the coefficients may look random and without any discernible patterns, but as we will see below they are actually not as random as they look.

All the expressions for $E$ above are quartic polynomials of $\partial_{i}$. Naively, one may estimate their size by $p^{4}$, where $p$ is the typical momentum scale in question. However, such an estimate is sloppy, since as an operator $p^{4}=\partial^{4}=\left(\partial^{2}\right)^{2}$, i.e., the square of the Laplacian operator, which is a scalar operator in $n$-dimensional Euclidean space, while $\mathcal{B}_{4}, \mathcal{B}_{22}$ and $\mathcal{A}_{31}$ are all non-scalar operators as defined. Thus motivated, we will define $E_{0}$, the scalar or isotropic part of $E$, as

$$
E_{0}=\int d \mu_{n-1} E=\text { (dimensionless numerical factor) } a^{2} \partial^{4}
$$

where $d \mu_{n-1}$ is again the unit measure on $S^{n-1}$. In other words, $E_{0}$ is $E$ integrated over all directions in $n$-dimensional momentum space. When one evaluates $E_{0}$, it turns out that

$$
E_{0}=\frac{1}{4 !} \partial^{4} a^{2} \times \begin{cases}6 /(n+2), & \text { for } Z_{n} \\ 12 /(n+2), & \text { for } D_{n} \text { or } A_{n}\end{cases}
$$

which is surprisingly simple (when contrasted with the expressions before the integration). Moreover, for all cases one has

$$
E_{0}=\rho^{2} \partial^{4} / 4(n+2)
$$

The above relation is not obvious (at least to the author) from the raw expressions for $E$, but once motivated it can actually be proved very easily for all lattices (not necessarily root lattices). Recall that $\Sigma$ is defined as

$$
\Sigma=\left\{\frac{1}{\tau} \sum_{X_{k} \in \mathcal{S}_{\rho}} \exp \left(X_{k} \cdot \partial\right)\right\}
$$

For each site $X_{k} \in \mathcal{S}_{\rho}$, one can expand the exponential as 


$$
\exp \left(X_{k} \cdot \partial\right)=1+\frac{1}{2 !}\left(X_{k} \cdot \partial\right)^{2}+\frac{1}{4 !}\left(X_{k} \cdot \partial\right)^{4}+\text { odd terms }+\mathcal{O}\left(\rho^{6}\right)
$$

where the odd terms will eventually be cancelled and do not concern us here. The quadratic and quartic terms can be rearranged in the following way.

$$
\begin{aligned}
& \left(X_{k} \cdot \partial\right)^{2}=\rho^{2} \partial^{2} / n+\left[\left(X_{k} \cdot \partial\right)^{2}-\rho^{2} \partial^{2} / n\right] \\
& \left(X_{k} \cdot \partial\right)^{4}=3 \rho^{4} \partial^{4} / n(n+2)+\left[\left(X_{k} \cdot \partial\right)^{4}-3 \rho^{4} \partial^{4} / n(n+2)\right] .
\end{aligned}
$$

The integrals

$$
\int d \mu_{n-1}\left(X_{k} \cdot \partial\right)^{2}=\rho^{2} \partial^{2} / n, \quad \int d \mu_{n-1}\left(X_{k} \cdot \partial\right)^{4}=3 \rho^{2} \partial^{4} / n(n+2),
$$

decree that the terms in square brackets in Eqs. (5.8) vanish under integration over all directions. Hence

$$
\int d \mu_{n-1} \Sigma=\frac{1}{\tau} \sum_{X_{k} \in \mathcal{S}_{\rho}}\left(1+\frac{1}{2 ! n} \rho^{2} \partial^{2}+\frac{3}{4 ! n(n+2)} \rho^{4} \partial^{4}\right)+\mathcal{O}\left(\rho^{6}\right),
$$

which is a sum over $\tau$ identical expressions and then divided by $\tau$, and is equal to the expression itself,

$$
\int d \mu_{n-1} \Sigma=1+\frac{1}{2 ! n} \rho^{2} \partial^{2}+\frac{3}{4 ! n(n+2)} \rho^{4} \partial^{4}+\mathcal{O}\left(\rho^{6}\right) .
$$

Lastly, we have the scalar part of $D^{2}$.

$$
\int d \mu_{n-1} D^{2}=\int d \mu_{n-1} 2 n(\Sigma-1) / \rho^{2}=\partial^{2}+\frac{6}{4 !(n+2)} \rho^{2} \partial^{4}+\mathcal{O}\left(\rho^{4}\right),
$$

and $E_{0}$ can be read off simply as the $\rho^{2}$ term.

The simple result Eq. (5.5) has important implications. Firstly, it gives us the following impossibility theorem:

One shell discretization of the Laplacian operator always suffers from order $a^{2}$ errors, regardless of the choice of the lattice.

One might have hope that, by using a lattice with a high degree of symmetry, all $\mathcal{O}\left(a^{2}\right)$ terms may vanish identically. We now see that is an impossible dream. There is always a 
scalar part which is not prohibited by any symmetry. The only way to eliminate order $a^{2}$ errors is by involving lattice sites which are not nearest neighbors, as we will discuss later in this paper.

Another corollary of Eq. (5.5) is that the scalar discretization error $E_{0}$ depends only on a single geometrical parameter - the packing diameter $\rho$. (It also depends on the spacetime dimensionality $n$, but that is usually determined by the physics problem in question.) One can then ask, in a given spacetime dimension, which lattice will minimize $E_{0}$ ? It will be the subject of the next section.

\section{ERROR DENSITIES OF ONE SHELL DISCRETIZATIONS}

As defined, the scalar discretization error $E_{0}$ is independent of how we describe the lattice on the coordinate space. This is certainly a pleasing feature to physicists, who know that all physical quantities should not depend on the choice of coordinates. On the other hand, $E_{0}$ is proportional to $\rho^{2}$, which is not surprising; the smaller $\rho^{2}$, the more lattice sites we are using to describe a given physical volume, and hence the smaller is the error. In other words, the error grows with $1 / \delta$, the volume of the unitcell of the lattice. To determine which class $\left(Z_{n}, D_{n}\right.$ or $\left.A_{n}\right)$ is optimal for our purposes, one defines the error density $\epsilon$ as the ratio of the $E_{0}$ to (a certain power of) the unitcell volume.

$$
E_{0}=\frac{1}{4(n+2)} \epsilon \delta^{-2 / n} \partial^{4} .
$$

The $-2 / n$ power has been chosen such that $\epsilon$ is $\rho$ independent; recall that $E_{0}$ and $\delta$ scale like $\rho^{2}$ and $\rho^{-n}$ respectively. Hence $\epsilon$ thus defined is a sheer parameter (not an operator) which just depends on which lattice which are using. For root lattices, it carries the value

$$
\epsilon= \begin{cases}1, & \text { for } Z_{n} \\ 2^{1-2 / n}, & \text { for } D_{n} \\ 2(n+1)^{-1 / n}, & \text { for } A_{n} .\end{cases}
$$


Obviously one can check that the error density of $Z_{n}$ is always smaller than that of $D_{n}$ and $A_{n}$. I] This is certainly not a surprising result, as we have shown in the previous section that $E_{0}$ is proportional just to $\rho^{2}$, and for our root lattices with the same $\rho, Z_{n}$ is the one with the smallest density.

We have seen that, among root lattices, $Z_{n}$ has the smallest error density. We can actually prove the much stronger result that $Z_{n}$ has the smallest error density among all lattices whose $\mathcal{S}_{\rho}$ have vanishing quadrapole moment, i.e., all the lattices with $D^{2}=\partial^{2}$ in leading order. The proof runs in two steps. First, observe that among all lattices whose basis vectors can be chosen to be nearest neighbors, $Z_{n}$ has the lowest density. (Among all parallelohedra with all sides of unit length, the one with the largest volume is a cube.) So $Z_{n}$ has the lowest error density among them. What about lattices whose basis vectors cannot all be chosen as nearest neighbors? Note that, for such a lattice $\Lambda$, there exists a proper sublattice $\bar{\Lambda} \subset \Lambda$ which is generated by the nearest neighbors of $\Lambda$. The lattices $\Lambda$ and $\bar{\Lambda}$ have the same $\rho$ and hence the same $E_{0}$, yet being a subset, the density of $\bar{\Lambda}$ is smaller than that of $\Lambda$. As a result, the error density of $\Lambda$ is larger than that of $\bar{\Lambda}$, which in turn by the previous argument is larger than that of $Z_{n}$. So we have the important result:

Under one shell discretization, the hypercubical lattices have the smallest error densities among all possible choices of lattices in the same number of spacetime dimensions.

Most lattice simulations are performed on hypercubical lattices, but usually the reasons are historical (following Wilson's example in Ref. [1]) or algorithmic ( $D^{2}$ can be easily written down as in Eq. (2.2) by using the trapezoidal rule). Here we provide another rationale: the hypercubical lattices have the lowest error density.

\footnotetext{
${ }^{9}$ Moreover, one also has $\epsilon_{Z_{2}}=\epsilon_{D_{2}}$ and $\epsilon_{A_{3}}=\epsilon_{D_{3}}$, i.e., the error density of a lattice is independent of how it is named.
} 


\section{ANISOTROPY}

While $E_{0}$ measures the isotropic part of the order $a^{2}$ errors, $E-E_{0}$ measures the anisotropy of $D^{2}$. The operator $E-E_{0}$ is a quartic polynomial in $\partial_{i}$, which in general can have all $\ell$-th multipole moment up to $\ell=4$. However, the first and third (dipole and octupole) multipole moments are identically zero as demanded by reflection symmetry, the second (quadrapole) moment is explicitly required to vanish, and the zeroth (monopole) moment $E_{0}$ has been subtracted out. So $E-E_{0}$ is purely of the fourth (hecadexapole) moment.

It is straightforward to verify that $E-E_{0}$ is non-vanishing for all root lattices with one exception: $D_{4}$, the checkerboard lattice in four dimensions.

$$
E=\frac{1}{4 !} 2 a^{2}\left(\mathcal{B}_{4}+\mathcal{B}_{22}\right)=\frac{2}{4 !} a^{2} \partial^{4}=E_{0} \quad \text { for } D_{4}
$$

That $D_{4}$ is more isotropic than $Z_{4}$ has been noted by Celmaster in Ref. [5], but here the result is stronger.

The checkerboard lattice in four dimensions $D_{4}$ is exactly isotropic at order $a^{2}$. It is the only unexceptional root lattice with this property.

One can understand the isotropy of the $D_{4}$ lattice by studying its geometry. The $D_{4}$ lattice has an accidental threefold discrete symmetry (which is also a symmetry of its Dynkin diagram) which mixes $\mathcal{B}_{4}$ and $\mathcal{B}_{22}$. The only combination which is invariant under this threefold symmetry is $\partial^{4}$. In other words, rotational symmetry is protected by this accidental threefold discrete symmetry at order $a^{2}$.

Since we are mainly interested in lattices in low dimensions $(n \leq 4)$, we have not studied the anisotropy of $E_{6,7,8}$, although the calculation should be straightforward (though tedious — the packing number $\tau$ is 240 for $E_{8}$ ). We also have not studied the issue of anisotropy for non-root lattices. However, since we have seen that the isotropy of $D_{4}$ is related to an

accidental discrete symmetry, a feature not apparent in these other cases, the author would be very surprised if other lattices share this property of isotropy. 


\section{BEYOND ONE SHELL DISCRETIZATION}

We have seen that order $a^{2}$ errors are unavoidable in one shell discretization. The order $a^{2}$ errors can be substantial on coarse lattices; for example, as a rule of thumb the order $a^{2}$ error on a hypercubical lattice is around $10 \%$ if $a$ is chosen to be around a third of the size of the physical system in question [21]. In order to reduce the error, one may try to use finer lattices. It is, however, a very costly procedure as the cost of lattice simulations grows at least as fast as the number of lattice sites, which is proportional to $a^{-n}$. (And that is before taking critical slowing down into account.) For $n=4$, to reduce the error to $1 \%$ would increase the cost a hundredfold. Another way of seeing the same result is by observing that the error density $\epsilon$, which is $a$ independent, is the proportionality constant between $E_{0}$ and $\delta^{-2 / n}$. To reduce $E_{0}$ by a factor of $10, \delta$ must be decreased by a factor of $10^{n / 2}$, i.e., a hundredfold in four dimensions.

Clearly it is not practical to control the $a^{2}$ errors just by using finer and finer lattices. Instead, one can completely eliminate the $a^{2}$ error by involving sites not in $\mathcal{S}_{\rho}$, i.e., by going beyond one shell discretization. As an example, we will review how this is done on hypercubical lattices.

Recall that, for hypercubical lattices, one shell discretization on $\mathcal{S}_{a}$ gives

$$
D^{2}\left[\mathcal{S}_{a}\right]=\mathcal{B}_{2}^{(n)}+\frac{2}{4 !} a^{2} \mathcal{B}_{4}^{(n)}+\mathcal{O}\left(a^{4}\right)=\partial^{2}+\frac{1}{12} a^{2} \sum_{1 \leq i \leq n} \partial_{i}^{4}+\mathcal{O}\left(a^{4}\right)
$$

The argument in the square brackets is to remind us of which shell we are using. One can also discretize on the following set of $2 n$ sites:

$$
2 \mathcal{S}_{a}=\{( \pm 2 a, 0, \ldots, 0)+\text { perm. }\}
$$

Notice that $2 \mathcal{S}_{a}$ is just $\mathcal{S}_{a}$ scaled up by a factor of 2 . As a result,

$$
D^{2}\left[2 \mathcal{S}_{a}\right]=\partial^{2}+\frac{1}{12}(2 a)^{2} \sum_{1 \leq i \leq n} \partial_{i}^{4}+\mathcal{O}\left(a^{4}\right)
$$

Consequently, the combination $\frac{4}{3} D^{2}\left[\mathcal{S}_{a}\right]-\frac{1}{3} D^{2}\left[2 \mathcal{S}_{a}\right]$ is free of order $a^{2}$ errors. 
Notice that $2 \mathcal{S}_{a}$ is not the second shell of $Z_{n}$. (In fact it is just part of the fourth shell.) Since the errors are in general proportional to some high powers of the radii of the shells involved, can we do better by using just the first and second shell, $\mathcal{S}_{a}$ and $\mathcal{S}_{\sqrt{2} a}$ ? Recall that $\mathcal{S}_{\sqrt{2} a}$ is the first shell of $D_{n}$, and $D^{2}$ has been calculated to be

$$
D^{2}\left[\mathcal{S}_{\sqrt{2} a}\right]=\mathcal{B}_{2}^{(n)}+\frac{2}{4 !} a^{2} \mathcal{B}_{4}^{(n)}+\frac{6}{4 !(n-1)} a^{2} \mathcal{B}_{22}^{(n)}+\mathcal{O}\left(a^{4}\right)
$$

which contains both $\mathcal{B}_{4}^{(n)}$ and $\mathcal{B}_{22}^{(n)}$. In contrast, $D^{2}\left[\mathcal{S}_{a}\right]$ involves just the former but not the latter. Thus one cannot obtain an expression free of both $\mathcal{B}_{4}^{(n)}$ and $\mathcal{B}_{22}^{(n)}$ errors by taking a linear combination of $D^{2}\left[\mathcal{S}_{a}\right]$ and $D^{2}\left[\mathcal{S}_{\sqrt{2} a}\right]$. In other words, one cannot eliminate the order $a^{2}$ order by taking a linear combination of $D^{2}$ of any two shells.

Bearing the above examples on the hypercubical lattices in mind, we will now move on to discuss the framework of elimination of the order $a^{2}$ errors on lattices in general. Consider the one shell discretization on two shells of radii $r_{1,2}$ with $r_{1}<r_{2}$. Then we want to see if the expression

$$
D^{2}\left[\mathcal{S}_{r_{1}}, \mathcal{S}_{r_{2}}\right]=c_{1} D^{2}\left[\mathcal{S}_{r_{1}}\right]-c_{2} D^{2}\left[\mathcal{S}_{r_{2}}\right]
$$

is equal to the Laplacian operator without any $a^{2}$ errors with some choices of coefficients $c_{1,2}$. Obviously in order to get the coefficient of the Laplacian operator to be unity we need $c_{1}-c_{2}=1$. We also want the scalar errors $E_{0}$ of the two shells, which are proportional to $r^{2}$, to cancel, by demanding $c_{1} r_{1}^{2}-c_{2} r_{2}^{2}=0$, or $c_{1} / c_{2}=\left(r_{1} / r_{2}\right)^{-2}$. Putting these together we have

$$
c_{1}=r_{2}^{2} /\left(r_{2}^{2}-r_{1}^{2}\right), \quad c_{2}=r_{1}^{2} /\left(r_{2}^{2}-r_{1}^{2}\right) .
$$

So now we can define the two shell discretization of the Laplacian operator over shells $\mathcal{S}_{r_{1}}$ and $\mathcal{S}_{r_{2}}$ as

$$
\begin{aligned}
D^{2}\left[\mathcal{S}_{r_{1}}, \mathcal{S}_{r_{2}}\right] & =\left(r_{2}^{2} D^{2}\left[\mathcal{S}_{r_{1}}\right]-r_{1}^{2} D^{2}\left[\mathcal{S}_{r_{2}}\right]\right) /\left(r_{2}^{2}-r_{1}^{2}\right) \\
& \equiv \partial^{2}+a^{2} \mathcal{H}_{2}+a^{4} \mathcal{H}_{4}+\mathcal{O}\left(a^{6}\right) .
\end{aligned}
$$


Thus defined, the scalar discretization error of order $a^{2}$ is guaranteed to vanish, i.e., $\int d \mu_{n-1} \mathcal{H}_{2}=0$. However, $\mathcal{H}_{2}$ itself may be non-zero, as shown by the example with the first and second shell in $Z_{n}$.

When we defined one shell discretization early in this paper, we had to explicitly demand that the shell in question have zero quadrapole moment. Here the situation is similar. For two shell discretization we have to demand that the two shell configuration in question, after weighting by $c_{1,2}$, has vanishing hexadecapole moment. In other words, the hexadecapole moments of the two shells must cancel each other.

In principle, one can translate the condition of the cancellation of hexadecapole moments into mathematical constraints and look for their most general solutions - but in practice it is almost impossible. (We have not even done that for the quadrapole case!) For example, for $Z_{n}$ or $D_{n}$, the shells described by $\left(y_{1}, \ldots y_{n}\right)$ and $\left(z_{1}, \ldots z_{n}\right)$ (plus permutation) will have their fourth multipole moment cancelled if and only if

$$
\sum_{i} y_{i}^{4}=K \sum_{i} z_{i}^{4}, \quad \text { and } \quad \sum_{i, j} y_{i}^{2} y_{j}^{2}=K \sum_{i, j} z_{i}^{2} z_{j}^{2} \quad \text { for some } K
$$

These are coupled diophantine-like constraints, which in general cannot be solved. The corresponding constraints for $A_{n}$ are even more hideous.

However, there are special cases which we know the hexadecapole moment are guaranteed to cancel, namely when the two shells are identical up to scaling (but no rotation). To be exact, let's define, for any natural number $p>1$,

$$
p \mathcal{S}_{\rho}=\left\{p X: X \in \mathcal{S}_{\rho}\right\}
$$

Then the two shell discretization $D^{2}\left[\mathcal{S}_{\rho}, p \mathcal{S}_{\rho}\right]$ has vanishing hexadecapole moment and hence is free of order $a^{2}$ errors. The proof is immediate. For $D^{2}\left[\mathcal{S}_{\rho}\right]=\partial^{2}+\rho^{2} \mathcal{H}_{2}+\mathcal{O}\left(a^{4}\right)$, one has

$$
\begin{aligned}
D^{2}\left[\mathcal{S}_{\rho}, p \mathcal{S}_{\rho}\right] & =\left((p \rho)^{2} D^{2}\left[\mathcal{S}_{\rho}\right]-\rho^{2} D^{2}\left[p \mathcal{S}_{\rho}\right]\right) /\left((p \rho)^{2}-\rho^{2}\right) \\
& =\left(p^{2} \rho^{2}\left(\partial^{2}+\rho^{2} \mathcal{H}_{2}\right)-\rho^{2}\left(\partial^{2}+p^{2} \rho^{2} \mathcal{H}_{2}\right)\right) /\left(p^{2}-1\right) \rho^{2}+\mathcal{O}\left(\rho^{4}\right) \\
& =\partial^{2}+\mathcal{O}\left(\rho^{4}\right) .
\end{aligned}
$$


In practice, the two shells should be chosen as small as possible to minimize the error. This suggests setting $p=2$, and

$$
D^{2}\left[\mathcal{S}_{\rho}, 2 \mathcal{S}_{\rho}\right]=\frac{4}{3} D^{2}\left[\mathcal{S}_{\rho}\right]-\frac{1}{3} D^{2}\left[2 \mathcal{S}_{\rho}\right]
$$

On the other hand, there is a unique case where the hexadecapole moments do cancel for two shells which are not related simply by scaling. As the reader may have guessed, this is the case for the first and second shells of the four dimensional checkerboard lattices, where the hexadecapole moment of each shell vanishes identically as dictated by the threefold discrete symmetry. Recall that both the first shell $\mathcal{S}_{\sqrt{2} a}$ and the second shell $\mathcal{S}_{2 a}$ contain 24 sites as described in Eqs. (4.13,4.14), and the two shell discretization over these two shells is

$$
D^{2}\left[\mathcal{S}_{\sqrt{2} a}, \mathcal{S}_{2 a}\right]=2 D^{2}\left[\mathcal{S}_{\sqrt{2} a}\right]-D^{2}\left[\mathcal{S}_{2 a}\right] \quad \text { for } D_{4},
$$

which can be checked to be free of order $a^{2}$ errors.

\section{ERROR DENSITIES OF TWO SHELL DISCRETIZATIONS}

Consider a two shell discretization with $a^{2}$ errors completely cancelled.

$$
D^{2}\left[\mathcal{S}_{r_{1}}, \mathcal{S}_{r_{2}}\right]=\partial^{2}+a^{4} \mathcal{H}_{4}+\ldots
$$

To study the order $a^{4}$ errors, one needs to know $\mathcal{H}_{4}$, which in general is a sextic polynomial of $\partial_{i}$. To calculate $\mathcal{H}_{4}$ would be a painful exercise. However, it is easy to evaluate $E_{0}^{\prime}$, the scalar part of $\mathcal{H}_{4}$,

$$
E_{0}^{\prime}=\int d \mu_{n-1} a^{4} \mathcal{H}_{4}
$$

Again we will start with the Taylor expansion of $\exp \left(X_{k} \cdot \partial\right)$.

$$
\exp \left(X_{k} \cdot \partial\right)=1+\frac{1}{2 !}\left(X_{k} \cdot \partial\right)^{2}+\frac{1}{4 !}\left(X_{k} \cdot \partial\right)^{4}+\frac{1}{6 !}\left(X_{k} \cdot \partial\right)^{6}+\text { odd terms }+\mathcal{O}\left(a^{8}\right)
$$

With $X_{k} \in \mathcal{S}_{r}$ (not necessarily the first shell), it can be rewritten as 


$$
\begin{aligned}
\exp \left(X_{k} \cdot \partial\right)=1 & +\frac{1}{2 !} I_{2} r^{2} \partial^{2}+\frac{1}{4 !} I_{4} r^{4} \partial^{4}+\frac{1}{6 !} I_{6} r^{6} \partial^{6} \\
& +[\ldots]+\text { odd terms }+\mathcal{O}\left(r^{8}\right)
\end{aligned}
$$

where $I_{l}$ is defined by

$$
\int d \mu_{n-1}\left(X_{k} \cdot \partial\right)^{l}=I_{l} r^{l} \partial^{l}
$$

with $I_{2}=1 / n$ and $I_{4}=3 / n(n+2)$. We have not calculated $I_{6}$ which fortunately is not needed for our purposes. Lastly, the [...] in Eq. (9.4), like the terms in square brackets in Eq. (5.8), vanishes under integration over all directions.

The scalar part of the one shell discretization on $\mathcal{S}_{r}$ is

$$
\int d \mu_{n-1} D^{2}\left[\mathcal{S}_{r}\right]=\partial^{2}+\frac{2 !}{4 !}\left(I_{4} / I_{2}\right) r^{2} \partial^{4}+\frac{2 !}{6 !}\left(I_{6} / I_{2}\right) r^{4} \partial^{6}+\mathcal{O}\left(r^{6}\right) .
$$

and the scalar part of the two shell discretization on $\mathcal{S}_{r_{1}}$ and $\mathcal{S}_{r_{2}}$ is

$$
D^{2}\left[\mathcal{S}_{r_{1}}, \mathcal{S}_{r_{2}}\right]=\partial^{2}-\frac{2 !}{6 !}\left(I_{6} / I_{2}\right) r_{1}^{2} r_{2}^{2} \partial^{6}+\mathcal{O}\left(r^{6}\right)
$$

and hence

$$
E_{0}^{\prime}=-\frac{2 !}{6 !}\left(I_{6} / I_{2}\right) r_{1}^{2} r_{2}^{2} \partial^{6}
$$

Now one can define the error density $\epsilon^{\prime}$ of a two shell discretization as

$$
E_{0}^{\prime}=-\frac{2 !}{6 !}\left(I_{6} / I_{2}\right) \epsilon^{\prime} \delta^{-4 / n} \partial^{6}
$$

As expected, using larger shells will increase the error density. For root lattices, and using shells $\mathcal{S}_{\rho}$ and $2 \mathcal{S}_{\rho}$, the error densities are

$$
\epsilon^{\prime}= \begin{cases}4, & \text { for } Z_{n} \\ 2^{4-4 / n}, & \text { for } D_{n} \\ 16(n+1)^{-2 / n}, & \text { for } A_{n}\end{cases}
$$

Again, the error densities are the smallest for hypercubical lattices. The reason is the same as the one shell case: for the same $\rho, Z_{n}$ has the lowest density. And the result can be as easily generalized to non-root lattices as before. 
On the other hand, for $D_{4}$ we can simply use the first and second shells, and the error density is

$$
\epsilon=4, \quad \text { for } \quad D^{2}\left[\mathcal{S}_{\sqrt{2} a}, \mathcal{S}_{2 a}\right],
$$

just as low as that of $Z_{n}$. Hence we come to the following conjecture:

Under two shell discretization, the hypercubical lattices have the smallest error densities among all possible choices of lattices in the same number of spacetime dimensions. The only

other case with an error density as low is two shell discretization on the first and second shells of the four dimensional checkerboard lattice.

The statement above is just a conjecture, not a theorem, as there may be two shells configurations other than $\mathcal{S}_{\rho}$ and $2 \mathcal{S}_{\rho}$ which have even lower error densities, just as the $D_{4}$ case we have seen. However, exact cancellations of the hexadecapole moments of two shells are very unlikely to happen without a symmetry rationale (like the threefold discrete symmetry of $D_{4}$ ), and since we do not see such extra symmetries in other lattices, the author concludes that it is very unlikely that counterexamples to the above conjecture will be found.

\section{CONCLUSION}

Let's recapitulate what we have learned through this study:

- One shell discretization faithfully reproduces the Laplacian operator (with order $a^{2}$ errors) if and only if the shell has vanishing quadrapole moment.

- The order $a^{2}$ error for one shell discretization is non-zero for any choice of lattice. The hypercubical lattices have the smallest error densities in any spacetime dimensions.

- Due to its threefold discrete symmetry, the four dimensional checkerboard lattice is isotropic up to order $a^{2}$. It is the only root lattice, and also probably the only lattice (root or non-root), with this property.

- Two shell discretization reproduces the Laplacian operator without order $a^{2}$ errors if and only if the hexadecapole moments of the two shells cancel each other. 
- The order $a^{2}$ error for one shell discretization is non-zero for any choice of lattice. It is likely (though not yet proved) that the hypercubical lattices have the smallest error densities in any spacetime dimensions. In four dimensions, the error density of the checkerboard lattice is just as small as the hypercubical.

One can see a clear pattern recurring throughout this discussion. The scalar (isotropic) properties of discretizations can be very cleanly studied by Taylor expanding $\exp \left(X_{k} \cdot \partial\right)$ and then integrating over all directions. By involving more shells, one can eliminate the errors order by order. On the other hand, the anisotropic properties are in general very difficult to study. The isotropy of $D_{4}$ is more like an accident (at least in our framework) - and it is straightforward to show that that the symmetry does not prevent higher multipoles appearing at order $a^{4}$. As a result, the most tractable way of eliminating anisotropic errors is by cancellation between shells related just by a simple scaling.

We have discussed the root lattices in detail and calculated their $\Sigma$ explicitly. However, most of our results are equally valid for root and non-root lattices. However, root lattices are so tractable in theory, and useful in practice, that it makes a very natural starting point. The only important non-root lattice in low dimensions is $D_{3}^{*}$, the three dimensional body-centered cubic lattice, which is the dual of $D_{3}$.

$$
D_{3}^{*} \equiv\left\{X=\left(x_{1}, x_{2}, x_{3}\right) \in a \mathbf{Z}^{3} \cup a\left(\mathbf{Z}^{3}+\left(\frac{1}{2}, \frac{1}{2}, \frac{1}{2}\right)\right)\right\},
$$

Its coordinates are either all integers or all half integers. Its nearest neighbors are $\left( \pm \frac{1}{2}, \pm \frac{1}{2}, \pm \frac{1}{2}\right)$, and angles between them are not rational fractions of $\pi$. One can study $D_{3}^{*}$ just as we study the root lattices, but we do not find any properties of interest.

What are the implications of this study for real lattice simulations? In no way does it challenge the canonical choice - the hypercubical lattice, which always has the smallest error density. The only case when one may consider otherwise is when isotropy is important; in such scenarios the four dimensional checkerboard lattice may be a tempting alternative. Still, as disclaimed in the introduction, the geometry of the lattice is only one of many factors which affect the efficiency of lattice simulations. Algorithmic considerations may overwhelm 
geometrical ones; remember the two shell discretization of $D_{4}$ involves 48 neighbors, opposed to 16 for $Z_{4}$. Still, the author expects this study is useful in providing a general framework in which different lattices may be discussed on equal footing, and the results may serve as "rules of thumb" for lattice simulations.

In this paper, we have studied how the geometries of non-hypercubical lattices affect the discretization of the Laplacian operator. Possible generalization to the case of the Dirac operator is possible but not straightforward, due to the problem of parity doubling. Without doing a careful analysis, it is difficult to foresee whether the results we have obtained in this paper can be extended to the Dirac case, However, the author is willing to make the following conjecture: the order $a^{2}$ discretization error of the Dirac operator does not vanish for any choice of lattice. Just as the discretization of the Laplacian operator is always plagued with errors like $a^{2} \partial^{4}$ which have the same tranformation properties as the Laplacian $\partial^{2}$, it is probable that the discretization of the Dirac operator $\gamma \cdot \partial$ will suffer from errors like

$a^{2}(\gamma \cdot \partial) \partial^{2}$. Hopefully the author can extend the present framework to the Dirac case in the near future and determine the correctness of this conjecture.

\section{ACKNOWLEDGMENTS}

I would like to thank Silas Beane, Tom Cohen, Jim Griffin and Steve Wallace for discussions. This work is supported by the U.S. Department of Energy grant DE-FG02-93ER40762. 


\section{REFERENCES}

[1] K.G. Wilson, Phys. Rev. D10 2445 (1974).

[2] N.H. Christ, R. Friedberg and T.D. Lee, Nucl. Phys. B202 89 (1982).

[3] N.H. Christ, R. Friedberg and T.D. Lee, Nucl. Phys. B210 310 (1982).

[4] N.H. Christ, R. Friedberg and T.D. Lee, Nucl. Phys. B210 337 (1982).

[5] W. Celmaster, Phys. Rev. D26 2955 (1982).

[6] W. Celmaster and F. Krausz, Phys. Rev. D28 1527 (1983).

[7] W. Celmaster, Phys. Rev. D28 2076 (1983).

[8] A. Di Giacomo, M. Maggiore and H. Panagopoulos, Phys. Rev. D36 2563 (1987).

[9] H. Neuberger, Phys. Lett. B199 536 (1987).

[10] G. Bhanot, K. Bitar, U.M. Heller and H. Neuberger, Nucl. Phys. B343 467 (1990).

[11] G. Bhanot, K. Bitar, U.M. Heller and H. Neuberger, Nucl. Phys. B353 551 (1991), Erratum B375 503 (1992).

[12] J.M. Drouffe and K.J.M. Moriarty, Nucl. Phys. B220 253 (1983) Erratum B226 547 (1983).

[13] R.W.B. Ardill, J.P. Clarke, J.M. Drouffe and K.J.M. Moriarty, Phys. Lett. B128 203 (1983).

[14] J.M. Drouffe and K.J.M. Moriarty, Z. Phys. C24 395 (1984).

[15] J.M. Drouffe, K.J.M. Moriarty and C.N. Mouhas, Comput. Phys. Commun. 30249 (1983).

[16] J.M. Drouffe, K.J.M. Moriarty and C.N. Mouhas, J. Phys. G10 115 (1984).

[17] W. Celmaster and K.J.M. Moriarty, Phys. Rev. D36 1947 (1987). 
[18] J.H. Conway and N.J.A. Sloane, "Sphere Packings, Lattices and Groups", SpringerVerlag (1992).

[19] R. Slansky, Phys. Rept. 791 (1981).

[20] H. Georgi, "Lie Algebras in Particle Physics", Benjamin-Cummings (1982).

[21] G.P. Lepage, Proceedings of TASI 1993, "The building blocks of creation" Boulder, 207 (1993). 